\title{
AMBARZUMYAN-TYPE THEOREMS ON STAR GRAPHS
}

\author{
Chuan Fu Yang, Vyacheslav N. Pivovarchik and Zhen You Huang
}

Abstract. The so-called Ambarzumyan theorem states that if the Neumann eigenvalues of the Sturm-Liouville operator $-\frac{d^{2}}{d x^{2}}+q$ with an integrable real-valued potential $q$ on $[0, \pi]$ are $\left\{n^{2}\right.$ : $n \geqslant 0\}$, then $q=0$ for almost all $x \in[0, \pi]$. In this work, the classical Ambarzumyan theorem is extended to star graphs with Dirac operators on its edges. We prove that if the spectrum of Dirac operator on star graphs coincides with for the unperturbed case, then the potential is identically zero.

Mathematics subject classification (2010): 34L05, 65L09.

Keywords and phrases: Inverse spectral problem, Dirac operator on star graphs, Ambarzumyan theorem.

\section{REFERENCES}

[1] V. A. Ambarzumyan, Über eine Frage der Eigenwerttheorie, Z. Phys., 53 (1929), 690-695.

[2] J. Bolte AND J. HARRISOn, Spectral statistics for the Dirac operator on graphs, J. Phy. A: Math. Gen., 36 (2003), 2747-2769.

[3] G. Borg, Eine Umkehrung der Sturm-Liouvilleschen Eigenwertaufgabe, Bestimmung der Differentialgleichung durch die Eigenwerte, Acta Math., 78 (1946), 1-96.

[4] G. BorG, Uniqueness theorems in the spectral theory of $y^{\prime \prime}+(\lambda-q(x)) y=0$, in Proc. 11th Scandinavian Congress of Mathematicians (Oslo: Johan Grundt Tanums Forlag), pp. 276-287, 1952.

[5] W. Bulla And T. Trenkler, The free Dirac operator on compact and noncompact graphs, J. Math. Phys., 31 (1990), 1157-1163.

[6] R. Carlson and V. N. PivovarchiK, Ambarzumian's theorem for trees, Electronic J. Diff. Equa. Vol. 2007, 142 (2007), 1-9.

[7] N. K. ChaKRaVARTY AND S. K. Acharyya, On an extension of the theorem of V. A. Ambarzumyan, Proc. Roy. Soc. Edinb. A, 110 (1988), 79-84.

[8] H. H. ChERN, C. K. LAW AND H. J. WANG, Extension of Ambarzumyan's theorem to general boundary conditions, J. Math. Anal. Appl., 309 (2005), 764-768.(corrigendum).

[9] H. H. Chern AND C. L. Shen, On the $n$-dimensional Ambarzumyan's theorem, Inverse Problems, 13 (1997), 15-18.

[10] S. CURRIE AND B. WATSOn, The M-matrix inverse problem for the Sturm-Liouville equation on graphs, Proc. Roy. Soc. Edinburgh Sect. A, 139, 4 (2009), 775-796.

[11] E. M. HARRELL, On the extension of Ambarzunyan's inverse spectral theorem to compact symmetric spaces, Amer. J. Math., 109 (1987), 787-795.

[12] M. Horváth, On a theorem of Ambarzumyan, Proc. Roy. Soc. Edinb. A, 131 (2001), 899-907.

[13] R. Kenyon, The Laplacian and Dirac operators on critical planar graphs, Invent. Math., 150 (2002), 409-439.

[14] M. KISS, An n-dimensional Ambarzumyan type theorem for Dirac operators, Inverse Problems, 20 (2004), 1593-1597.

[15] V. Kostry Kin and R. Schrader, Kirchoff's rule for quantumn wires, J. Phys. A: Math. Gen., 32 (1999), 595-630.

[16] T. Kottos And U. Smilans Ky, Quantum chaos on graphs, Phys. Rev. Lett. , 79 (1997), 4794-4797.

[17] T. KotTOS AND U. SMILANSKY, Periodic orbit theory and spectral statistics for quantum graphs, Ann. Phys., 247 (1999), 76-124. 
[18] P. Kuchment, Quantum graphs: an introduction and a brief survey, pp. 291-314, in Analysis on Graphs and its Applications, Proc. Symp. Pure. Math., AMS 2008.

[19] N. V. KuZNeTsov, Generalization of a theorem of V. A. Ambarzumian, Dokl. Akad. Nauk SSSR, 146 (1962), 1259-1262 (in Russian).

[20] C. K. Law And V. N. Pivovarchik, Characteristic functions of quantum graphs, J. Phys. A: Math. Theor., 42 (2009), 035302(11pp).

[21] B. M. LEVITAN AND M. G. GAS YMOV, Determination of a differential equation by two of its spectra, Usp. Mat. Nauk, 19 (1964), 3-63.

[22] B. M. Levitan And I. S. SARgSJan, Sturm-Liouville and Dirac Operators (Russian), Nauka, Moscow 1988: English transl., Kluwer, Dordrecht, 1991.

[23] V. N. Pivovarchik, Ambarzumyan's theorem for a Sturm-Liouville boundary value problem on a star-shaped graph, Funct. Anal. Appl., 39 (2005), 148-151.

[24] C. L. SHEN, On some inverse spectral problems related to the Ambarzumyan problem and the dual string of the string equation, Inverse Problems, 23 (2007), 2417-2436.

[25] C. F. YANG AND Z. Y. HUANG, Inverse spectral problems for $2 m$-dimensional canonical Dirac operators, Inverse Problems, 23 (2007), 2565-2574.

[26] C. F. YANG AND X. P. YANG, Some Ambarzumyan-type theorems for Dirac operators, Inverse Problems, 25 (2009), 095012(13pp).

[27] V. A. YURKO, Inverse spectral problems for Sturm-Liouville operators on graphs, Inverse Problems, 21 (2005), 1075-1086. 